Referencia para citar este artículo: Bianchi, E. (2016). Diagnósticos psiquiátricos infantiles, biomedicalización y DSM: ¿hacia una nueva (a)normalidad? Revista Latinoamericana de Ciencias Sociales, Niñez y Juventud, 14 (1), pp. 417-430.

\title{
Diagnósticos psiquiátricos infantiles, biomedicalización y DSM: ¿hacia una nueva (a) normalidad?*
}

\author{
EUGENIA BIANCHI ${ }^{* *}$ \\ Profesora Universidad de Buenos Aires, Argentina.
}

\begin{abstract}
Artículo recibido en mayo 15 de 2015; artículo aceptado en julio 21 de 2015 (Eds.)
\end{abstract}
- Resumen (analítico): Presento resultados de una investigación postdoctoral que aborda el análisis de las transformaciones recientes en los diagnósticos en salud mental infantil, a través del estudio de dos categorías: TDAH (Trastorno por Déficit de Atención e Hiperactividad) y TEA (Trastorno del Espectro Autista).

Empleando métodos analítico-interpretativos sobre bibliografia especifica, recupero y sistematizo tópicos trabajados desde perspectivas de la medicalización y la biomedicalización, y estudios sobre el riesgo en salud mental, para el análisis de las tipificaciones psiquiátricas mencionadas.

Como un aporte a estos campos, desarrollo la hipótesis según la cual las reformulaciones acaecidas en el manual de clasificación psiquiátrica DSM-5 en el TDAHy TEA, pueden entenderse a la luz de las características más amplias de los procesos de medicalización y biomedicalización que recaen con énfasis en las infancias.

Palabras clave: diagnóstico, infancia, biomedicalización, riesgo, TDAH, autismo, DSM (Tesauro Isoc de Psicología).

\section{Psychiatric diagnoses of children, biomedicalization and DSM: towards a new (ab)normality?}

- Abstract (analytical): This article presents the results from postdoctoral research that addresses recent transformations in the diagnosis of children's mental health through the analysis of two categories: ADHD (Attention Deficit Hyperactivity Disorder) and ASD (Autism Spectrum Disorder).

Using analytical and interpretative methods taken from a specialist bibliography, the author recovers and systematizes topics addressed from the perspectives of medicalization and biomedicalization. Studies on risks to mental health are also used for the analysis of the psychiatric classifications analysis already mentioned.

As a contribution to these fields, the author develops the hypothesis that the changes made to the DSM-5 psychiatric classification manual, specifically for ADHD and ASD can be understood in the light of the broader processes of medicalization and biomedicalization that have a particular focus on childhood.

Este artículo corto forma parte de la investigación postdoctoral en curso "Transformaciones recientes en los diagnósticos en salud mental infantil en Argentina (1994-2015). De trastornos mentales, saberes profesionales y manuales psiquiátricos: las infancias normalizadas en el siglo XXI". El estudio sigue lineamientos de investigación cualitativa, Área de conocimiento: Sociología (temas especiales: estudios de infancia y niñez; salud mental y ciencias sociales). Es financiado con Beca Postdoctoral Conicet, Resolución Decreto Nacional 2926; 8/8 2014 Conicet. Ministerio de Ciencia, Tecnología e Innovación Productiva de la República Argentina, período junio 2014-junio 2016.

** Licenciada en Sociología, Magíster en Investigación en Ciencias Sociales y Doctora en Ciencias Sociales, Universidad de Buenos Aires. Profesora de grado y posgrado Universidad de Buenos Aires. Becaria Postdoctoral II.GG.-Conicet. Correo electrónico: eugenia.bianchi@yahoo.com.ar 
Key words: diagnosis, childhood, biomedicalization, risk, ADHD, autism, DSM (Thesaurus Isoc Psychology).

\section{Diagnósticos psiquiátricos infantis, biomedicalização e DSM: para uma nova (a)normalidade?}

- Resumo (analítico): Apresenta-se os resultados de uma pesquisa de pós-doutorado que aborda a análise das transformações recentes nos diagnósticos de saúde mental infantil por meio do estudo de duas categorias: TDAH (Transtorno de Déficit de Atenção e Hiperatividade) e TEA (Transtorno do Espectro do Autismo).

Utilizando métodos analíticos e interpretativos sobre literatura específica, o artigo recupera e sistematiza temas trabalhados a partir de perspectivas de medicalização e biomedicalização e de estudos sobre o risco de saúde mental para a análise das caracterizações psiquiátricas mencionadas.

Como contribuição a esses campos, se desenvolve a hipótese de acordo com as reformulações ocorridas no manual de classificação psiquiátrica DSM-5 em TDAH e TEA e como elas podem ser entendidas à luz das características mais amplas dos processos de medicalização e biomedicalização que têm ênfase na infância.

Palavras-chave: diagnóstico, infância, biomedicalização, risco, TDAH, autismo, DSM (Tesauro Isoc Psicologia).

-1. Introducción. -2. El diagnóstico como objeto de estudio. -3. Infancia, diagnósticos y salud mental. -4. Infancia y biomedicalización en el siglo XXI: riesgo y diagnósticos. -5. Riesgo, manuales de clasificación e infancia. -6. TEA, TDAH y sus transformaciones en el DSM. -6a. EI riesgo en el TEA: Dimensiones, espectro y gradualidad. -6b. TDAH: la detección precoz y el riesgo a futuro. -7 . Consideraciones finales. -Lista de referencias.

\section{Introducción}

De la mano de las transformaciones en la biomedicina y la psiquiatría biológica en el siglo XXI, los diagnósticos en salud mental infantil han experimentado sustanciales transformaciones. En particular, dos categorías psiquiátricas ofrecen elementos para analizar estos cambios: TDAH (Trastorno por Déficit de Atención e Hiperactividad) y TEA (Trastorno del Espectro Autista). El artículo analiza estos cambios a partir de dos perspectivas conexas: por una parte, los estudios sobre la medicalización y biomedicalización de la sociedad; y por otra, los trabajos acerca del riesgo en salud mental. Emplea para ello métodos analíticointerpretativos sobre bibliografía específica, con el objetivo de realizar aportes a estos campos de estudios. A este fin, se desarrolla la hipótesis según la cual las reformulaciones producidas en el DSM-5 para el TDAH y TEA pueden inscribirse en las transformaciones más amplias de los procesos de medicalización y biomedicalización enfocados en las infancias.
La relevancia de estos aportes reside en que habilitan la reflexión acerca de modalidades novedosas y específicas de normalización derivadas del accionar conjunto de los saberes de la salud mental infantil.

\section{EI diagnóstico como objeto de estudio}

El diagnóstico médico en general, y de los trastornos mentales en particular, se constituyó como objeto de reflexión desde diferentes perspectivas, incluidas la filosofía, la epistemología, la sociología de la ciencia y del conocimiento, los estudios sociales de la ciencia, y la antropología médica crítica. Foucault inscribió el diagnóstico en el estudio de los regímenes de veridicción (Foucault, 1991, 2007, 2009) es decir el conjunto de reglas que permiten establecer -en una sociedad y en relación a un cierto discurso- cuáles son los criterios de verdad, que permiten decidir acerca de la veracidad o falsedad de ciertos enunciados y formulaciones. Analizó tanto el 
diagnóstico psiquiátrico (Foucault, 2005) como médico-clínico (Foucault, 2003) en el marco de estrategias de los Estados capitalistas para gestionar poblaciones, impulsadas como parte de la medicalización de la sociedad (Foucault, 1996).

En los últimos dos siglos, el rol del diagnóstico en las prácticas médicas se reconfiguró, incrementando progresivamente su carácter técnico, especializado y burocrático (Rosenberg, 2002). Desde el último tercio del siglo XIX, las categorías diagnósticas se expandieron, incorporando aspectos como las emociones, la idiosincrasia y la conducta culturalmente disruptiva (Rosenberg, 2005). El acceso a imágenes del cerebro, y la interacción entre las neurociencias, la genética y la biología molecular, y las intervenciones neuroquímicas y quirúrgicas, dotan al proceso de diagnóstico $\mathrm{y}$ tratamiento de herramientas novedosas e insoslayables (Vidal, 2009).

El Manual de Diagnóstico y Estadísticas de los Trastornos Mentales (Diagnostic and Statistical Manual of Mental Disorders, DSM) suscita debates por el diagnóstico en salud mental. El arco que va desde su giro hacia la psiquiatría biológica en la versión III de 1980, pasando por la penetración global de la versión IV de 1994, hasta la publicación de la versión 5 en 2013, opera como eje sobre el que gravitan parte de las discusiones en torno al diagnóstico desde distintos saberes de la salud mental que se disputan al niño como objeto y como problema (Bianchi, 2015b).

Rose (2012) estudió el diagnóstico en relación a los cambios en las racionalidades y tecnologías de gobierno, para pensar las políticas de la vida en el siglo XXI. Enfocando en los procesos de medicalización de la sociedad, se estudiaron además la expansión de los diagnósticos en el siglo XXI (Conrad \& Potter, 2003, Rose, 2006, 2012) y la incidencia de actores científicos, médicos, industriales, políticos, empresarios, entre otros; entablando relaciones de fuerza en juego estratégico, así como condiciones de posibilidad para la emergencia de las diversas categorías (Conrad, 2005, 2007, Conrad \& Leiter, 2004, Rose, 2012).
Según Rose (1996) en virtud de las transformaciones en la biomedicina, el diagnóstico psiquiátrico tiene hoy un contenido médico parcial, incluyendo además otras condiciones relacionadas con el desenvolvimiento social, como el historial escolar, de empleos, la vida afectivo-familiar, la capacidad de compra y manejo del dinero, y también información sobre la conducta pasada y el comportamiento peligroso.

Caponi (2009) examinó las implicancias epistemológicas de los diagnósticos, analizando cómo diferentes aspectos de la clasificación de depresión se validan y legitiman. Good (2003) trabajó el modo en el que la medicina construye sus objetos, considerando la relación entre cultura, enfermedad y conocimiento médico, y tomando a la construcción de la enfermedad como objeto de diagnóstico y de actividad terapéutica. Y Hacking (1999) incluyó una serie de variables en el análisis de los efectos de las clasificaciones sobre los sujetos clasificados, que abarcan personas, clasificaciones, expertos, instituciones y conocimientos, y que permiten diferenciar entre la inclusión y la exclusión en una clasificación.

Un desprendimiento reciente de la perspectiva de la medicalización lo constituyen los estudios de la biomedicalización (Clarke, Mamo, Fishman, Shim \& Fosket, 2003, Clarke $\&$ Shim, 2011), que en este artículo se analiza en relación a la noción de riesgo presente en los diagnósticos de TDAH y TEA. También reciente es el enfoque en la globalización de los diagnósticos medicalizados (Conrad \& Bergey, 2014).

\section{Infancia, diagnósticos y salud mental}

El estudio de los diagnósticos psiquiátricos en la infancia tiene estatuto propio, ya que constituye el segmento poblacional más intensamente gobernado (Rose, 1998), y es uno de los blancos dilectos de múltiples estrategias de normalización individual y colectiva en las sociedades occidentales (Donzelot, 1998, Rose, 1998, Foucault, 2001).

Además de vincularla a la reproducción de la población, Scheper-Hughes y Sargent (1998) incluyen entre las funciones de la infancia, a 
aspectos culturales de personalidad, moralidad, orden social y trastornos, congregando discursos y prácticas sobre sexualidad, reproducción, amor y protección, poder y autoridad, y sus potenciales abusos.

Con la modernidad, la niñez se posiciona como foco de estrategias orientadas a promover activamente algunas capacidades y atributos (inteligencia, educación, estabilidad emocional, entre otras). La salud, el bienestar y las características de crianza de los niños se ligaron al destino de la nación y a las responsabilidades del Estado. Durante los siglos XIX y XX, los proyectos para preservarla modelaron detalles de la vida doméstica, conyugal y sexual de sus padres. Estos proyectos se complementaron con acciones directas sobre los cuerpos infantiles, para impartir conocimiento, inculcar conductas o supervisar, evaluar y rectificar patologías (Rose, 1999).

Daroqui y López (2012) inscriben la historia de las políticas de intervención sobre la infancia, en procesos más abarcativos de control y normalización social, en los que la niñez conforma un continuum con otros subconjuntos poblacionales considerados peligrosos $\mathrm{O}$ en riesgo, en una serie constituida también por locos, salvajes, criminales, proletarios y animales (Varela \& Álvarez-Uría, 1991, Castel, 1986). La infancia constituyó, como ellos, un sector problemático de la sociedad, y su existencia supuso una amenaza presente o futura para el bienestar del Estado, sea bajo la figura del delincuente potencial que amenazaba la propiedad y la seguridad, como futuro trabajador cuyas habilidades y moral debían ser moldeadas, o como futuros soldados que debían mantener un estado físico óptimo para luchar en nombre de la Nación (Rose, 1999). Esta serie se gobernó mediante estrategias diversas, desde abiertamente penales, a tutelares y asistenciales, abarcando "tecnologías de cura, corrección, represión, protección, disciplinamiento, segregación o, en sus extremos, incapacitación y eliminación" (Daroqui \& López, 2012, p. 49).

El cuidado de la infancia se gestionó bajo la modalidad tutelar, y se expresó como corrección, tratamiento y psicologización (Donzelot, 1998). La tutela se orientó a la protección, socorro y educación del niño desamparado, a la corrección del niño difícil, $\mathrm{y}$ al castigo del futuro delincuente, intentando mitigar ciertas potencialidades, tanto en lo referido a la falta de productividad, como a la beligerancia con normas establecidas, que podían tornarse revolucionarias (Huertas, 2005). Establecida como saber normativo por excelencia, la medicina (primordialmente la psiquiatría, la pediatría y la neurología) colaboró con la criminología y la pedagogía en las tareas de diagnosticar, clasificar y tratar a la "infancia degenerada", con objetivos y resultados disímiles (Rossi, 2003).

El siglo XIX resignificó el encierro de los residuos disciplinarios (Foucault, 2005), aquellos imposibles de clasificar, distribuir, ordenar, asimilar, educar o reducir. Las prácticas de segregación en sus diferentes variantes, complementaron $\mathrm{y}$ dieron sentido a otras modalidades de ejercicio del poder que inciden en los sujetos hasta hoy (Huertas, 2009).

Aunque como marcan Daroqui y López, se trata de "reprimir para separar, separar para curar, para disciplinar, para proteger, para corregir, para incapacitar, para neutralizar" (Daroqui \& López, 2012, p. 50), cada época exhibe especificidades en la estructura económica, política y cultural, que permean los saberes expertos para el diagnóstico de colectivos asociados a la disfuncionalidad en relación a pautas de normalización y conductas esperadas. Conceptos como desamparo, vicio, mala vida, peligrosidad, promiscuidad, riesgo, carencia o vulnerabilidad, y e instrumentos jurídicos como "riesgo moral o material" o "interés superior del niño", expresan imaginarios y representaciones heterogéneos acerca de quienes requieren las intervenciones específicas.

\section{Infancia y biomedicalización en el siglo XXI: riesgo y diagnósticos}

El estudio de diagnósticos como el TDAH y el TEA, habilita el análisis de algunas características del proceso de medicalización hoy, y el papel que en él cumple la infancia. Los estudios de la medicalización llevan más de cinco décadas de existencia, incluyendo aportes desde perspectivas como la sociología médica, la historia, la antropología, la salud pública, la 
economía, la bioética y la literatura (Conrad, 2013). Originalmente orientada en la expansión del dominio médico hacia áreas previamente no consideradas en esa clave, este enfoque se reconfiguró, abarcando hoy un espacio complejo de inteligibilidad que contempla la definición, descripción, comprensión y tratamiento de un problema en términos médicos (Conrad, 2007), e incluyendo el análisis de modos de saber y verdad asociados al conocimiento científicotecnológico.

De cara a las transformaciones de la biomedicina, el cuidado médico y la psiquiatría biológica, en el siglo XXI se incorporaron a las perspectivas de la medicalización, aportes de las nociones de gubernamentalidad (Foucault, 2006) y de biosociabilidad (Rabinow, 1996), generando la denominada biomedicalización.

La gubernamentalidad remite a actividades orientadas a moldear, guiar, conducir o afectar las conductas de las personas, y se ubica en la articulación de las tecnologías de dominación de los otros, y las tecnologías del yo, referidas a uno mismo (Foucault, 1990). La noción de biosociabilidad, por su parte, expresa nuevas formas de grupos sociales, comunidades e identidades conformadas en torno a un cierto rasgo biológico, como una variación genética específica, o una clasificación biomédica (Rabinow, 1996).

Clarke et al. (2003) sistematizaron algunos ejes con los cuales ubican diferencias entre los estudios de la medicalización, y la corriente de la biomedicalización. Entre ellos, el foco que la biomedicalización tiene en la salud, el riesgo y la vigilancia, una tríada que ya estaba presente en los procesos de medicalización, pero que en los fenómenos analizados por Clarke y sus colegas adquiere características específicas.

A diferencia de la medicalización, dirigida a la enfermedad, la biomedicalización no restringe su accionar a procesos mórbidos, sino que apunta a la salud misma (Clarke, Mamo, Fosket, Fishman \& Shim, 2010). Esto se cristaliza en que, además de encargarse de la definición, detección y tratamiento de enfermedades, configura complejos conglomerados de factores abstractos e indicios cuya co-ocurrencia produce un riesgo. Este cambio de eje inaugura nuevas fórmulas de gestión de poblaciones, enmarcadas en modos de gobierno específicos. Castel (1986) entiende esta mutación como el paso de una clínica del sujeto a una clínica epidemiológica, sosteniendo que el "riesgo no es el resultado de un peligro concreto del que es portador un individuo o incluso un grupo determinado, sino que es un efecto de la correlación de datos abstractos o factores que hacen más o menos probable la materialización de comportamientos indeseables" (Castel, 1986, p. 229).

Los datos generales, impersonales, pueden interrelacionarse y reagrupar factores heterogéneos entre sí. Como remarca Rose (1996) estos factores no necesariamente son peligrosos individualmente; es la agrupación lo que dispara el riesgo. La lógica misma del "estar en riesgo", por un lado prescinde de la manifestación de síntomas específicos, y por otro instaura una gradación de la ocurrencia de la enfermedad, antes que la presencia o ausencia de la misma (Bianchi, 2012), más propia de la dinámica de la medicalización.

Clarke y Shim (2011) sostienen que los procesos de medicalización y biomedicalización no se reemplazan, sino que coexisten espaciotemporalmente. Los tópicos clásicos de la medicalización no se extinguen; se reformulan y son incluidos en procesos de biomedicalización.

Rose, O’Malley y Valverde (2006) sostienen que el riesgo no designa una realidad fenoménica, sino un modo de concebir y tratar problemas. Como técnica probabilística, el riesgo clasifica numerosos eventos en una distribución de casos. Los valores que arroja esa distribución constituyen insumos para realizar predicciones que reduzcan daños. Y aunque la técnica del riesgo es abstracta, da lugar a una miríada de formas concretas de gobierno.

La dinámica del riesgo consiste en que la detección de un conjunto de factores desencadena una señal. Sin embargo, es una existencia probabilística y abstracta de riesgos, el punto de partida no es un observable, sino una situación deducida a partir de riesgos que la configuran como tal. Las estrategias de gobierno basadas en la lógica del riesgo promueven modalidades de vigilancia sistemática, a fin de anticipar e impedir un suceso no deseable o una dificultad, sea una enfermedad, una anomalía, 
un comportamiento desviado, la pérdida de trabajo o la criminalidad, entre otras.

\section{Riesgo, manuales de clasificación e infancia}

Las perspectivas de la medicalización y biomedicalización dan cuenta de la centralidad del riesgo como elemento de análisis de las transformaciones de la biomedicina en el siglo XXI. El riesgo como matriz utilizada en el gobierno de poblaciones, y en especial como modalidad de creciente uso en la salud mental infantil (Grinberg, 2008, Bianchi, 2015a), se patentiza de formas diferentes en diagnósticos específicos.

En este marco, cobran relevancia los manuales de clasificación de los denominados trastornos psiquiátricos. Mundialmente se destacan la Clasificación Internacional de Enfermedades y Problemas de Salud (CIE, en inglés ICD, International Classification of Diseases), y el DSM. La CIE es un manual de diagnóstico en salud pública elaborado por la Organización Mundial de la Salud (OMS, en inglés WHO, World Health Organization), a diferencia del DSM que es editado por una asociación privada de psiquiatras estadounidenses, la American Psychiatric Association (APA).

El primero es una nosografía descriptiva del conjunto de los problemas de saludenfermedad, y la inclusión de un apartado para problemáticas de salud mental data de la sexta edición, de 1952. Ese mismo año se publica la primera edición del DSM, que comparte con el CIE la orientación epidemiológica y los fines estadísticos (Faraone, 2013).

La tercera versión del DSM, publicada en 1980, supone una transformación epistemológica y tecnológica capital, iniciando un proceso de penetración mundial del manual en la práctica clínica psiquiátrica. Esta transformación incluyó rasgos que se consolidaron en versiones subsiguientes, entre ellas: flexibilidad, dinamismo, estandarización, a-teoricidad, prescindencia de explicaciones etiológicas, y sustento en la observación de sintomatología conductual, generando numerosas críticas del campo de la salud mental (Bianchi, 2014).

El DSM no es el manual de diagnóstico de trastornos mentales más utilizado en el mundo. La encuesta mundial realizada en 2011 por la Asociación Psiquiátrica Mundial (WPA, World Psychiatric Association) y la OMS, arrojó que el $70,1 \%$ de los psiquiatras encuestados emplea la décima versión vigente de la CIE. Sin embargo, la mayoría de los psiquiatras que no utilizan la CIE refirió emplear el DSM (Reed, Correia, Esparza, Saxena \& Maj, 2011). En su análisis de la medicalización del TDAH como fenómeno globalizado, Conrad y Bergey (2014) señalan que la psiquiatría estadounidense está en franca expansión, y remarcan que una consecuencia de este fenómeno es el uso cada vez más extendido del DSM, en oposición a los criterios de la CIE.

La expansión para los diagnósticos infantiles, estrictamente hablando, no se inaugura con el DSM-5. Según Frances (2014) presidente del grupo de trabajo de la versión anterior del manual, ya en aquella publicación se desarrollaron sobrediagnósticos de cuadros como autismo, TDAH y la denominada bipolaridad infantil.

El DSM-5 elaboró tipificaciones como el TDAH y el TEA en las que se identifican factores de riesgo que no coinciden exactamente con el trastorno que se manifestaría a futuro, sino que estas tipificaciones pincelan una condición psiquiátrica en el presente que es diferente de aquella sobre la cual pesa el riesgo futuro, y que reviste menor severidad en la actualidad. Los problemas metodológicos suscitados por estos constructos incluyen, entre otros aspectos, que “a) la mayoría de las personas con 'factor de riesgo' finalmente no desarrollan la enfermedad temida; b) la mayoría de las personas con 'factor de riesgo' son indistinguibles de los 'normales'; c) actualmente no hay intervenciones eficaces para evitar un inevitable estigma de rotulación en psiquiatría" (Heerlein, 2013, p. 22).

La prevención derivada de identificar factores de riesgo pretende construir las condiciones objetivas de aparición del peligro, para deducir de ellas nuevas y múltiples modalidades de intervención, y lograr el control absoluto de lo imprevisto (Castel, 1986). En este contexto, la publicación del DSM-5, con los 
cambios mencionados, reposiciona a la infancia como blanco eminentemente gobernable, al que los procesos de biomedicalización tienen entre sus objetivos dilectos.

Sin embargo, esta lógica del riesgo opera de modo diferencial en las dos tipificaciones mencionadas. Esquemáticamente, en el TDAH el riesgo se relaciona con los futuros posibles de los niños que han sido diagnosticados, $\mathrm{y}$ con el presente de los adultos que no fueron diagnosticados; y el riesgo en el TEA se expresa por el énfasis en la gradualidad con la que se asume que puede manifestarse el cuadro.

\section{TEA, TDAH y sus transformaciones en el DSM}

Tanto el diagnóstico de TDAH como el de TEA presentan elementos relacionados con la lógica del riesgo. Sin embargo, ésta no se pone en juego del mismo modo en ambas tipificaciones. Esto es consonante con los procesos biomedicalizadores, que no trazan líneas, sino que anudan actores, estrategias, tecnologías, saberes y prácticas de modos cambiantes y no unitarios.

La publicación del DSM-5 en mayo de 2013 supone una reactualización de controversias, a la luz de la incorporación, tanto de nuevas nomenclaturas como de nuevos criterios diagnósticos. Ya desde la publicación de los borradores del DSM-5 (http://www. dsm5.org) previo a su lanzamiento definitivo, la arquitectura y contenidos del manual suscitaron posicionamientos críticos desde múltiples frentes analíticos y clínicos (Frances, 2009, 2014, Malta-Oliveira, 2012, Caponi, 2014).

Los cambios en el DSM-5 impactan en varios aspectos: la reducción de los subtipos "no especificados" de trastornos; la clasificación dimensional y su mixtura con el paradigma categorial, centrando la definición del trastorno en la intensidad de su expresión, y no en presencia o ausencia de síntomas (con la consecuente multiplicación de comorbilidades); la desaparición o reagrupamiento de categorías diagnósticas poco precisas; y la definición de síndromes de riesgo (manifestaciones leves o mild) para prevenir trastornos graves que si no son detectados pueden desarrollarse en la adultez (APA, 2013).

La tipificación inmediatamente anterior al actual TEA (en inglés Autism Spectrum Disorder, ASD) fue el TGD (en inglés Pervasive Developmental Disorder, PDD) definido en el DSM-IV. Se dividía en cinco categorías, e incluía como nosologías predominantes al Síndrome Autista y Síndrome de Asperger, junto con el Síndrome de Rett, Trastorno Desintegrativo de la infancia (o autismo secundario), y Trastorno Generalizado del Desarrollo No Especificado (APA, 1994).

El derrotero conceptual del Síndrome Autista y el Síndrome de Asperger se inicia en 1943, cuando Leo Kanner describe los disturbios autísticos del contacto afectivo, o autismo infantil (Zappella, 1998). Hasta los '80 el autismo era diagnosticado como esquizofrenia infantil (APA, 1980), siendo diferenciado de esta con posterioridad (APA, 1994). En 1944 Asperger describe la psicopatía autística. En 1981, Wing sistematiza estas descripciones, renombrando el cuadro como Síndrome de Asperger (Attwood, 1998). Propone también la noción de Espectro Autista, ubicando al autismo como extremo más severo, y al Asperger como el más leve, aunque compartan bases estructurales y neurobiológicas (Attwood, 2006). Esta formulación derivó en la inclusión de ambos cuadros en un grupo común en el DSM, el TGD.

El DSM-5 no reconoce los diferentes subtipos que conformaban al TGD. En su lugar, divide a los TEA en dos categorías: Trastorno de la Comunicación Social, y Patrones repetitivos de Comportamiento, Intereses y Actividades. Los criterios diagnósticos incluyen antecedentes conductuales y comportamientos actuales. El nivel de severidad ocupa un lugar destacado, englobando en un mismo conjunto de dificultades del desarrollo a las limitaciones sociales y de la comunicación, y la presencia de patrones comportamentales repetitivos $\mathrm{y}$ restringidos (APA, 2013).

Por su parte, el TDAH (en inglés Attention Deficit Hyperactivity Disorder, o ADHD), tiene varios momentos históricos. En 1902 Still describe niños con inatención significativa, que afecta el rendimiento escolar, y lo asocia 
con un defecto mórbido en el control moral (Barkley, 2006b). En la década del '20 surge la concepción neurológica del trastorno, de la que se desprende el concepto de Strauss de lesión cerebral mínima, de origen presuntamente infeccioso (Lange, Reichl, Lange, Tucha \& Tuch, 2010). La imposibilidad de detectarlo y las críticas a esta concepción llevaron a que en 1962 el cuadro se denominara disfunción cerebral mínima (Strother, 1973, Barkley, 2006a). En 1971 Wender integra la hiperactividad, el trastorno de atención y percepción, las dificultades de aprendizaje, y la impulsividad y trastornos afectivos (Lakoff, 2000). En 1980 el DSM-III incorpora el diagnóstico del síndrome de déficit de atención (APA, 1980). El DSM III-R une déficit de atención e hiperactividad (APA, 1987). El DSM-IV-TR distingue tres subtipos: con predominio de inatención, con predominio de hiperactividad-impulsividad, y combinado (APA, 2000).

En el DSM-5, la tipificación del TDAH mantiene la estructura en torno a síntomas y subtipos, aunque añade aclaraciones ampliatorias de la importancia en cada síntoma. Además, eleva la edad de manifestación de algunos síntomas de 7 a 12 años. También cobra relevancia el diagnóstico de TDAH en adultos. Se contempla además la comorbilidad entre TEA y TDAH, e incluyen índices de severidad (APA, 2013).

\section{6 a. El riesgo en el TEA: Dimensiones, espectro y gradualidad}

El DSM-5 procura profundizar su aproximación a una estrategia dimensional para clasificar los diagnósticos. Este enfoque se distancia de la orientación categorial de las dos versiones precedentes, inclinadas al énfasis en la presencia o ausencia de una lista de síntomas, mayormente observables conductuales. En el DSM-5, el enfoque dimensional "se logra combinando categorías con diferente severidad, en una sola categoría con indicadores dimensionales de severidad" (Heerlein, 2013, p. 22).

La combinación de factores es una característica saliente de la lógica del riesgo. Foucault analizó este aspecto, considerando la noción de caso, como una modalidad de saber acerca de la enfermedad con dos vectores: por un lado apunta a individualizar el fenómeno colectivo de la enfermedad, y por otro busca integrar los fenómenos mórbidos individuales en un campo colectivo. Estas dos líneas se despliegan calculando y cuantificando eventualidades, desde una aproximación racional e identificable. Una enfermedad se concibe como una distribución de casos cuando se toma en cuenta una población espaciotemporalmente circunscripta, sobre la que se realizan análisis cuantitativos, y se calculan eventualidades de muerte o contagio. Esto permite establecer riesgos puntuales para individuos o poblaciones, que pueden ser positivos o negativos, es decir, tanto de contagio (morbilidad) o muerte (mortalidad), como de no-contagio o cura (Foucault, 2006).

Estos riesgos pueden calcularse "para cada individuo, según su edad, el lugar donde viva, y lo mismo para cada categoría de edad, cada ciudad, cada profesión" (Foucault, 2006, p. 81). El análisis de riesgo en términos de distribución de casos establece diferentes intensidades de riesgo. Las especificidades individuales, etarias, de localización u otras condiciones, instauran áreas de mayor o menor riesgo, formulándose riesgos diferenciales, que identifican las características más peligrosas.

El diagnóstico de TEA reformulado en el DSM-5 ilustra esta lógica clasificatoria que apela a la combinación de categorías con diferente severidad. El DSM-5 reserva el trastorno por autismo para designar los casos más severos, $\mathrm{y}$ el trastorno por Asperger para los menos severos. En lugar de clasificar por división de categorías, las diferentes tipificaciones dentro de los TEA se organizan en relación a una gradación de severidad (Heerlein, 2013).

Un corolario epistémico de la noción de espectro, y de la gradualidad en la tipificación diagnóstica, es que la diferencia tajante entre normalidad y psicopatología se diluye. Según Paris, "la convicción de que el trastorno mental es un punto en un continuum se desprende directamente de un modelo dimensional" (Paris, 2013, p. 41).

Este desplazamiento hacia un modelo dimensional conlleva que la ampliación 
de espectros se asocia a una ampliación de diagnósticos. Este punto fue identificado y analizado por Conrad y Potter (2003), quienes estudiaron la extensión del TDAH, históricamente caracterizado como un diagnóstico primordialmente infantil, y su desplazamiento para incluir crecientemente a adultos, y abordaron este fenómeno en el marco de considerar que las categorías diagnósticas que han sido absorbidas por los procesos de medicalización, una vez establecidas, pueden expandirse y tornarse más inclusivas. Afines argumentaciones desarrollan Clarke etal.(2003), sosteniendo que esta expansión de diagnósticos se relaciona con que la biomedicalización se orienta a la salud misma, y un efecto de esta expansión es el incremento en las posibilidades de detección de falsos positivos.

Volviendo al autismo, Paris (2013) subraya que la amplitud del concepto de trastorno del espectro autista descripto en el DSM5 resulta en una ampliación respecto de la tipificación clínica clásica del cuadro. Esta nueva clasificación, menos restrictiva, abre a interpretaciones de acuerdo con las cuales algunas excentricidades individuales podrían ser releídas a la luz de este diagnóstico.

Un último elemento a considerar reside en que el tratamiento no incluye, al menos de modo predominante, una terapéutica psicofarmacológica, como sí ocurre en el TDAH (Lange et al., 2010, Barkley, 2006b). Para quienes son diagnosticados con autismo, esta particularidad habilita posibilidades tanto de estigmatización, como de conformación de identidades en términos de identificación con la enfermedad (Paris, 2013), en lo que algunos investigadores analizaron empleando el concepto de sujeto cerebral (Ehrenberg, 2009, Ortega, 2009, Ortega \& Vidal, 2007).

Otra novedad del DSM-5 es la categorización simultánea (Vasen, 2015). Las comorbilidades cruzadas de los TEA con otros trastornos en los que la terapéutica farmacológica sí es predominante, como el TDAH, abren la posibilidad de una expansión de estas terapéuticas, inaugurando nuevos riesgos por polimedicación.

\section{6 b. TDAH: la detección precoz y el riesgo a futuro}

Otra línea respecto de las lógicas del riesgo en la clasificación de los denominados trastornos mentales en el DSM-5 se abre a partir de las modificaciones realizadas al TDAH. El DSM-5 está orientado hacia la detección precoz y la prevención de los denominados trastornos mentales. Uno de sus objetivos es que su aplicación para efectuar los diagnósticos permita realizar la detección temprana y la intervención de los factores de riesgo en la prevención de enfermedades futuras (Heerlein, 2013).

De hecho, la lógica del riesgo supone un trastocamiento de las concepciones psiquiátricas y epidemiológicas clásicas acerca de la prevención e intervención en salud mental. Las modalidades de intervención inscriptas en los procesos de biomedicalización no tienen la orientación clásica de la intervención, hacia la reforma, corrección, castigo o cura de un individuo concreto, sino que se enfocan a la correlación estadística de elementos heterogéneos susceptibles de producir un riesgo (Castel, 1986). Al escindirse el peligro de la manifestación de los síntomas, el centro queda en la constatación de particularidades, que aquellos considerados como especialistas han instituido en factores de riesgo (Rose, 1998).

En el caso del TDAH, sin embargo, la integración argumentativa y clínica entre la lógica del riesgo, la prevención, la intervención, y la consideración de factores de riesgo respecto de un padecimiento futuro adquiere ribetes particulares. Una de las consecuencias de la ampliación de las categorías diagnósticas derivada del despliegue de procesos de medicalización de la sociedad, consiste en el caso del TDAH, en el sostenido incremento del diagnóstico en adultos (Conrad \& Potter, 2003, Batstra \& Frances, 2012). De hecho, el TDAH en el DSM-5 ha dejado de considerarse un trastorno de inicio en la infancia, adolescencia y juventud, para incluirse como un trastorno del neurodesarrollo, abriendo la posibilidad de un diagnóstico en la adultez (APA, 2013).

Un motor para este incremento en el diagnóstico en adultos viene dado por la 
posibilidad de ubicar aspectos de la vida cotidiana como factores de riesgo. En especial en el TDAH se identifican dos tipos de factores de riesgo, que habilitan a considerar al TDAH como un diagnóstico presente en la infancia, o que siendo diagnosticado en la adultez, puede rastrearse en los años de infancia. Por un lado, aquellos factores que suponen una alta comorbilidad con otras patologías psiquiátricas (depresión, trastornos de alimentación, consumo de sustancias psicoactivas y trastorno de ansiedad); y por otro aquellas dificultades manifiestas para el desenvolvimiento en la vida social (delincuencia, fracaso escolar o académico, dificultades familiares, afectivas o laborales) (Bianchi, 2015a).

Sobre las comorbilidades, Scandar (2009) listó el trastorno de personalidad antisocial y trastorno de abuso de drogas psicoactivas, trastornos de ansiedad y trastornos de humor.

La lógica del riesgo en el TDAH también opera en las argumentaciones que aluden a disímiles factores, cuya interacción establece trayectorias a futuro. Algunos de los factores que integran esas trayectorias posibles suponen un incremento de riesgos, y otros conducen a morigerarlos. Así, en función de la integración de factores, por un lado se establece un perfil de mayor probabilidad de consumo de sustancias ilegales asociado al TDAH en la adolescencia, especialmente si no se realiza el diagnóstico. Pero por otro lado la trayectoria derivada de ese perfil puede modificarse si en la interacción de factores seincluye laincidencia de la prescripción de fármacos legales, como el metilfenidato, sobre el que se documentaron cuantiosos efectos adversos y contraindicaciones (Mayes, Bagwell \& Erkulwater, 2008), disminuyendo las probabilidades de consumo de sustancias ilegales (Bernaldo de Quirós, 2000). Esta configuración constituye una paradoja, porque para erradicar un riesgo (el consumo de drogas), se generan nuevos riesgos (la administración de metilfenidato).

Sobre las dificultades futuras para el desenvolvimiento social, en el TDAH estas se manifestarían en la vida académica, laboral, social y afectiva, constituyendo nuevos riesgos, susceptibles a su vez de nuevas políticas preventivas, que incluyen tanto peligros internos al individuo como amenazas externas inmanejables (Castel, 2004). Para el TDAH abarcan cambios permanentes de intereses, inconsecuencia laboral, cambios en la vida en pareja y las tareas hogareñas, empleos en puestos de baja calificación, dificultades académicas y financieras, automedicación, consumo de drogas y alcohol, accidentes automovilísticos y exacerbación de fantaseos y ensoñaciones (Scandar, 2009, Tallis, 2007).

Rose sostiene que ya los términos del juicio psiquiátrico no son clínicos, y ni siquiera epidemiológicos como consideró Castel, sino ligados a la gestión de lo cotidiano. La falta de habilidad para afrontar a la familia, el estudio, el trabajo, el dinero, las labores domésticas; todos son, potencialmente, criterios de calificación psiquiátrica, porque marcan fallas en la "administración del yo" (Rose, 1998), autorizando el accionar de prácticas divisorias, que separan el yo que puede encargarse de sí mismo, del yo que -dado que no puede afrontar las dificultades de la vida cotidiana- debe ser administrado por otros (Rose, 1996).

\section{Consideraciones finales}

Las dinámicas de configuración de la díada normal-anormal fueron ejes centrales de las estrategias de gobierno de poblaciones e individuos inauguradas con la conformación de los Estados capitalistas. En el núcleo de esas estrategias, la medicalización introdujo una torsión, por la cual lo anormal se asimiló a lo patológico (Huertas, 2009). En este desplazamiento, los diagnósticos psiquiátricos tienen un rol destacado para la atribución de parámetros de normalidad y anormalidad, y la infancia representa uno de los segmentos sobre el cual la normalización resulta de múltiples vectores de disciplinas y proyectos.

Analizadas desde la medicalización en el siglo XXI y la biomedicalización, las transformaciones recientes en saberes, tecnologías y métodos de ejercicio de psiquiatría biológica -de la que el manual DSM-5 constituye un instrumento fundamental- introdujeron una nueva torsión en la díada, modificando las coordenadas de comprensión de la misma con diversas características que se resumen, como 
marcan Clarke et al. (2003), en enfocarse en la salud misma.

Dichas transformaciones incluyen la disolución de una diferencia tajante, discreta, entre normalidad y patología, reposicionando las cuestiones de grado, de intensidad sintomática dimensional, antes que la presencia o ausencia de marcadores biológicos o síntomas para diagnosticar el cuadro. Modeladas además por la lógica del riesgo y la prevención, la clínica de lo cotidiano y la expansión de los diagnósticos psiquiátricos, estas transformaciones se caracterizan por no circunscribirse a los polos de salud y enfermedad. Los polos permanecen, pero las intervenciones actúan en el presente para asegurar el estado óptimo y el mejor futuro posible de los sujetos (Rose, 2012). En este objetivo de optimización, se busca no sólo revelar estas patologías invisibles lo antes posible, sino intervenir en ellas, aun en un estado de presintomaticidad.

En el siglo XXI, los cambios de la biomedicina y de la psiquiatría biológica no evidenciaron intenciones de abolir ni prescindir de la normalización, sino que la han modelado con características particulares, reconfigurando los límites de la normalidad y la patología, de la enfermedad mental y la salud mental (Rose, 2006). Los estudios de la medicalización y biomedicalización, con matices y convergencias analíticas, ofrecen herramientas para aproximarse críticamente a configuraciones estratégicas inauguradas con la publicación del DSM-5, evidenciando que la normalización no es un concepto cerrado, ni circunscripto históricamente, y que pueden identificarse formas de normalización no vinculadas a la idea de recta, de desorden o desvío en torno a una media.

Diagnósticos como el TDAH y el TEA ilustran que la normalización puede estar inserta también en una lógica de modulación, donde la norma a alcanzar se deduce de un juego de distribuciones diferenciales (Foucault, 2006), y no de un estándar único ni previo de normalidad, y que los parámetros contra los que se realizan las operaciones de normalización responden a una distribución puntual de factores.

\section{Lista de referencias}

APA (1980). Diagnostic and Statistical Manual of Mental Disorders Third Edition. Washington: APA.

APA (1987). Diagnostic and Statistical Manual of Mental Disorders Third Edition-Revised. Washington: APA.

APA (1994). Diagnostic and Statistical Manual of Mental Disorders. Fourth Edition. Washington: APA.

APA (2000). Diagnostic and Statistical Manual of Mental Disorders. Fourth Edition. Text Revision. Washington: APA.

APA (2013). Diagnostic and Statistical Manual of Mental Disorders, Fifth Edition. Washington: APA.

Attwood, T. (1998). Asperger's syndrome: a guide for parents and professionals. London \& Philadelphia: Jessica Kingsley Publishers.

Attwood, T. (2006). Complete Guide to Asperger's Syndrome. LondonPhiladelphia: Jessica Kingsley Publishers.

Barkley, R. (2006a). Attention-Deficit Hyperactivity Disorder. A handbook for diagnosis and treatment. New York: The Guilford Press.

Barkley, R. (2006b). The relevance of the Still Lectures to Attention-Deficit/Hyperactivity Disorder. A Commentary. Journal of Attention Disorders, 10 (2), pp.137-140. Doi: $10.1177 / 1087054706288111$

Batstra, L. \& Frances, A. (2012). DSM-5 further inflates attention deficit hyperactivity disorder. Journal of Nervous and Mental Disease, 200, pp. 486-488. Doi: 10.1097/ NMD.0b013e318257c4b6

Bernaldo de Quirós, G. (2000) ¿Medicar o no medicar? Revista Terremotos y Soñadores, 1, pp. 10-13.

Bianchi, E. (2012). El problema del riesgo. Notas para una reflexión sobre los aportes surgidos de la usina genealógica en torno al concepto de riesgo en salud mental. Espacios Nueva Serie, 1, pp. 84-97.

Bianchi, E. (2014). Todo tiene un principio... y en el principio fue el DSM-III. El desbloqueo epistemológico y tecnológico 
de la psiquiatría biológica estadounidense. CulturasPsi, 2 (1), pp. 87-114.

Bianchi, E. (2015a). "El futuro llegó hace rato". Susceptibilidad, riesgo y peligrosidad en el diagnóstico y tratamiento por TDAH en la infancia. Revista de Estudios Sociales 2 (52), pp. 185-199. Doi: http://dx.doi. org/10.7440/res52.2015.13

Bianchi, E. (2015b) Infancia, normalización y salud mental. Figuras históricas y encadenamientos actuales en la formulación del ADHD (Trastorno por Déficit de Atención e Hiperactividad). Historia, Ciências, Saúde-Manguinhos, 22 (3), pp. 761-779. Doi: http://dx.doi. org/10.1590/S0104-59702015000300006.

Caponi, S. (2009). Un análisis epistemológico del diagnóstico de depresión. InterfaceComunicação, Saúde, Educação, 29, pp. 327-338. Doi: http://dx.doi.org/10.1590/ S1414-32832009000200007

Caponi, S. (2014). O DSM-V como dispositivo de segurança. Physis Revista de Saúde Coletiva, 24 (83), pp. 741-763. Doi: http://dx.doi.org/10.1590/S010373312014000300005.

Castel, R. (1986). De la peligrosidad al riesgo. En F. Álvarez-Uría \& J. Varela (comps.) Materiales de Sociología crítica, (pp. 219243). Madrid: La Piqueta.

Castel, R. (2004). La inseguridad social ¿Quées estar protegido? Buenos Aires: Manantial.

Clarke, A., Mamo L., Fishman J., Shim, J. \& Fosket J. (2003). Biomedicalization: Technoscientific Transformations of Health, Illness and U. S. Biomedicine. American Sociological Review, 68 (2), pp. 161-194. Doi: http://www.jstor.org/ stable/1519765

Clarke, A., Mamo L., Fosket J., Fishman J. \& Shim, J. (2010). Biomedicalization. Technoscience, Health and Illness. Durham-London: Duke University Press.

Clarke. A. \& Shim, J. (2011). Medicalization and Biomedicalization Revisited: Technoscience and Transformations of Health, Illness and American Medicine. En B. Pescosolido, J Martin, J. McLeod \& A. Rogers (eds.) Handbook of the Sociology of Health, Illness, and Healing. A Blueprint for the 21st Century, (pp.173-200). New York-Dordrecht-Heidelberg-London: Springer.

Conrad, P. (2007). The medicalization of society. On the transformation of human conditions into treatable disorders. Baltimore: The John Hopkins University Press.

Conrad, P. (2013). Medicalization: Changing Contours, Characteristics, and Contexts. En W. Cockerham (ed.) Medical Sociology on the move. New directions in theory, (pp. 195-214). Dordrecht-Heilderberg-New York-London: Springer.

Conrad, P. \& Bergey, M. (2014). The impending globalization of ADHD: Notes on the expansion and growth of a medicalized disorder. Social Science and medicine, 122, pp. 31-43. Doi: http://dx.doi.org/10.1016/j. socscimed.2014.10.019

Conrad, P. \& Leiter, V. (2004). Medicalization, markets and consumers. Journal of Health and Social Behavior, 45, pp. 158-176.

Conrad, P. \& Potter, D. (2003). From hyperactive children to ADHD adults. Observations on the expansion of medical categories. En P. Conrad \& V. Leiter (eds.) Health and health care as social problems, (pp. 3965). Lanham-Boulder-New York-TorontoOxford: Rowman \& Littlefield Publishers Inc.

Conrad, P. (2005). The shifting engines of medicalization. Journal of Health and Social Behavior, 46, pp. 3-14.

Daroqui, A. \& López, A. (2012). Introducción. En A. Daroqui, A. López \& R. F. CiprianoGarcía (comps.) Sujeto de castigos. Hacia una sociología de la penalidad juvenil, (pp. 49-60). Santa Fe: Homo Sapiens.

Donzelot, J. (1998). La policía de las familias. Valencia: Pre-Textos.

Ehrenberg, A. (2009). O sujeito cerebral. Psicologia. Clinica, Rio de Janeiro, 21 (1), pp. 187-213. Doi: http://dx.doi. org/10.1590/S0103-56652009000100013

Faraone, S. (2013). Reformas en salud mental. Dilemas en torno a las nociones, conceptos y tipificaciones. Salud Mental y Comunidad-UNLa, 3, pp. 29-40. 
Foucault, M. (1990). Tecnologías del yo y otros textos afines. Barcelona: Paidós.

Foucault, M. (1991). Microfísica del poder. Madrid: La Piqueta.

Foucault, M. (1996). La vida de los hombres infames. Buenos Aires: Altamira.

Foucault, M. (2001). Los Anormales. Buenos Aires: Fondo de Cultura Económica.

Foucault, M. (2003). Nacimiento de la Clínica. Buenos Aires: Siglo XXI.

Foucault, M. (2005). El Poder Psiquiátrico. Buenos Aires: Fondo de Cultura Económica.

Foucault, M. (2006). Seguridad, territorio, población. Buenos Aires: Fondo de Cultura Económica.

Foucault, M. (2007). Nacimiento de la biopolítica. Buenos Aires: Fondo de Cultura Económica.

Foucault, M. (2009). El gobierno de sí y de los otros. Buenos Aires: Fondo de Cultura Económica.

Frances, A. (2009). Whither DSM-V? British Journal of Psychiatry, 195, pp. 391-392. Doi: 10.1192/bjp.bp.109.073932

Frances, A. (2014). ¿Somos todos enfermos mentales? Manifiesto contra los abusos de la psiquiatría. Barcelona: Ariel.

Good, B. (2003). Medicina, racionalidad $y$ experiencia. Una perspectiva antropológica. Barcelona: Bellaterra.

Grinberg, J. (2008). Transformaciones en el tratamiento de la niñez en riesgo. Reflexiones sobre un dispositivo de protección a la infancia en la ciudad de Buenos Aires. Cuadernos de Antropología Social, 27, pp. 155-174.

Hacking, I. (1999). The social construction of what? Cambridge-Massachusetts-London: Harvard University Press.

Heerlein, A. (2013). Aproximaciones al DSM5: ¿un avance en psiquiatría? Vertex Revista Argentina de Psiquiatría, XXV, pp. 18-26.

Huertas, R. (2005). La medicalización de la delincuencia infantil en la España del primer tercio del siglo XX. En M. Miranda \& G. Vallejo (comps.) Darwinismo social y eugenesia en el mundo latino, (pp. 375400). Buenos Aires: Siglo XXI.
Huertas, R. (2009). Medicina social, control social y políticas del cuerpo. La subjetivación de la norma. En M. Miranda \& A. Girón-Sierra (coords.) Cuerpo, Biopolítica y Control Social, (pp. 19-42). Buenos Aires: Siglo XXI.

Lakoff, A. (2000). Adaptive will: The evolution of Attention Deficit Disorder. Journal of the history of the behavioral sciences, 36 (2), pp. 149-169. Doi: 10.1002/(SICI) 1520$6696(200021) 36: 2<149:: A I D$ JHBS3>3.0.CO;2-9

Lange, K., Reichl, S., Lange, K., Tucha, L. \& Tucha, O. (2010). The history of attention deficit hyperactivity disorder. $A D H D$ Attention Deficit Hyperactivity Disorders, 2, pp. 224-255. Doi: 10.1007/s12402-0100045-8

Malta-Oliveira, S. (2012). Os alcances e limites da medicalização do risco para a psicose: a emergência de uma nova categoria? Physis Revista de Saúde Coletiva, 22 (1), pp. 291309. Doi: http://dx.doi.org/10.1590/S010373312012000100016

Mayes, R., Bagwell, C. \& Erkulwater, J. (2008). ADHD and the Rise in Stimulant Use Among Children. Harvard Review of Psychiatry, 16 (3), pp. 151-166. Doi: 10.1080/10673220802167782

Ortega, F. (2009). The Cerebral Subject and the Challenge of Neurodiversity. BioSocieties, 4, pp. 425-445. Doi: http://dx.doi. org/10.1017/S1745855209990287

Ortega, F. \& Vidal, F. (2007). Mapping the cerebral subject in contemporary culture. Electronic Journal of Communication Information \& Innovation in Health, 1 (2), pp. 255-259. Doi: 10.3395/reciis.vli2.90en

Paris, J. (2013). The ideology behind DSM-5. En J. Paris \& J. Phillips (eds.) Making the DSM-5. Concepts and controversies, (pp. 39-46). New York-Heilderberg-DordrechtLondon: Springer.

Rabinow, P. (1996). Essays on the Anthropology of Reason. New Jersey: Princeton University Press.

Reed, G., Correia, J., Esparza, P., Saxena, S. \& Maj, M. (2011). The WPA-WHO Global Survey of Psychiatrists' Attitudes Towards 
Mental Disorders Classification. World Psychiatry, 10, pp. 118-131.

Rose, N. (1996). The death of the social? Re-figuring the territory of government. Economy and Society, 25 (3), pp. 327-356. Doi: 10.1080/03085149600000018

Rose, N. (1998). Inventing our selves. Psychology, power and personhood. Cambridge: Cambridge University Press.

Rose, N. (1999). Governing the Soul. The shaping of the private self. London-New York: Free Association Books.

Rose, N. (2006). Disorders without borders? The Expanding Scope of Psychiatric Practice. BioSocieties, 1, pp. 465-484. Doi:10.1017/S1745855206004078

Rose, N. (2012). Políticas de la vida. Biomedicina, poder y subjetividad en el siglo XXI. Buenos Aires: Unipe.

Rose, N., O' Malley, P. \& Valverde, M. (2006). Governmentality. Annual Revievof Law and Social Science, 2, pp. 83-104. Doi: 10.1146/ annurev.lawsocsci.2.081805.105900

Rosenberg, Ch. (2002). The tyranny of diagnosis. Specific entities and individual experience. The Milbank Quarterly, 80 (2), pp. 237-260. Doi:10.1111/1468-0009.t01$1-00003$

Rosenberg, Ch. (2005). Contested boundaries. Psychiatry, disease and diagnosis. Perspectives in Biology and Medicine, 49 (3), pp. 407-424. Doi:10.1353/ pbm.2006.0046

Rossi, G. (2003). La locura en los niños hacia finales del siglo XIX en Buenos Aires. Primera parte. Dos tesis precursoras presentadas ante la Facultad de Medicina porteña. Revista Temas de Historia de la Psiquiatría Argentina, 17, pp. 1-14.

Scandar, R. (2009). El niño que no podía dejar de portarse mal. TDAH: Su compresión y tratamiento. Buenos Aires: Distal.

Scheper-Hughes, N. \& Sargent, C. (1998). Small Wars. The cultural politics of childhood. Berkeley: University of California Press.

Strother, Ch. (1973). Minimal cerebral dysfunction: a historical overview. Annals of the New York Academy of Sciences, 1 (205), pp. 6-17.
Tallis, J. (2007). Neurología y trastorno por déficit de atención: mitos y realidades. En B. Janin (comp.) Niños desatentos e hiperactivos. ADD/ADHD. Reflexiones críticas acerca del Trastorno por Déficit de Atención con o sin Hiperactividad, (pp. 187-223). Buenos Aires: Noveduc.

Varela, J. \& Álvarez-Uría, F. (1991). Arqueología de la escuela. Madrid: La Piqueta.

Vasen, J. (2015). Autismos: ¿espectro o diversidad? Familias, maestros $y$ profesionales ante el desafio de repensar etiquetas. Buenos Aires: Noveduc.

Vidal, F. (2009). "Brainhood, anthropological figure of modernity". History of the Human Sciences, 22 (1), pp. 5-36. Doi: 10.1177/0952695108099133

Zappella, M. (1998). Autismo infantil: Estudios sobre la afectividad y las emociones. México, D. F.: Fondo de Cultura Económica. 